\title{
Investigation of Cyclic Electrolytic Solutions Injection in Clay-Containing Oil Reservoirs
}

\section{Kadet VV and Chagirov PS*}

Gubkin Russian State University of Oil and Gas, Russian Federation

\begin{abstract}
Analysis of osmotic and ion exchange processes at the interface between injected agent and clay-containing rock has been carried out. The analysis allowed to obtain reservoir properties depending on ionic composition of the injected water and clay-content of reservoir. It's been shown that decrease of salinity of the injected agent leads to the transformation of the structure of the porous medium: share of «thin» pores increases, whereas the share of «thick» pores reduces, resulting in reduced porosity. Analytical relative permeabilities depending on the salt concentration and ion composition of injected fluid have been obtained on the basis of micromechanical description of fluid flow process in frame of the lattice model of porous medium. The modeling of two-phase Newtonian fluids flow in clay-containing porous medium has been carried out. The influence of parameters of porous medium, injected saline fluid also injection frequency on efficiency of oil displacement has been analyzed.
\end{abstract}

Keywords: Electrolityc solution; Low-salinity; Water flooding; Percolation modeling

\section{Nomenclature}

$R_{0}$ - the hydration rate of the mineral in a dilute electrolyte solution

$\mathrm{C}_{0}$ - ion concentration in fresh water

$\mathrm{C}_{\mathrm{Mel}}$ - cation exchange capacity of clays

$C_{\mathrm{Me} 2}$ - concentration of electrolytic solution in fluid,

$\mathrm{C}_{\mathrm{Me2 \Gamma}}$ - concentration of substituting cations

$I_{\mathrm{c}}$ - ionic strength of the electrolyte

$A$ - characteristic constant of the solvent

$K_{e q}$ - the equilibrium constant of the cation exchange reaction

$K_{c l}$ - clayiness index

$m_{0}-$ initial porosity

$f(r)$ - porometrical function

$S_{r}$ - share of adsorbed water in rock

$F\left(S, C_{M e 2}\right)-$ Buckley-Leverett function

$q$ - unit discharge

$P_{k}\left(S, C_{M e 2}\right)$ - capillary pressure function

$M_{i}\left(S, C_{M e 2}\right)$ - phase mobility

$R_{k}$ - radius of the reservoir external boundary

$r_{w}-$ well radius

$D^{*}-$ coefficient taking into account diffusion and hydrodynamic dispersion

\section{Introduction}

Interest in the tertiary method of oil field development, called LSW (Low Salinity Waterflooding) [1], grows in recent years. This method implies obtaining additional oil by fresh water injection into the reservoir. In some experiments on different cores [1-3] an increase of displacement efficiency has been recorded. Among the conditions under which described effect occurs:

- A large share of clay minerals in the rock;

- Produced water contains divalent cations $\mathrm{Ca}^{2+}$ or $\mathrm{Mg}^{2+}$;

- Injected water has a reduced concentration of salts in comparison with the formation water.

It is known that hydrochemical imbalance in the «solution-rock» system causes change in clay mineral volume under the influence of osmotic and ion exchange processes, which significantly affects the properties of the clay-containing reservoirs. In addition, changing the hydrochemical regime of reservoir may lead to change in the type of clay mineral, entailing the destruction of molecular structure of clay and its disaggregation [4]. Besides the salt concentration in the water, hydration of clay minerals is also dependent on the composition of the dissolved salts.

\section{Analysis of osmotic and ion exchange processes at the contact of clay mineral and electrolyte solution}

The swelling value and swelling nature of clays are determined by several factors, majority of them are mineral composition of injected fluid and the structure and composition of clay minerals . Clay minerals with a hard crystal structure (e.g. kaolinite) have the least swelling value, minerals with unsteady crystal structure (e.g. montmorillonite) united by a common name smectites [5] have the largest swelling value. Interaction with fresh water in smectites causes the osmotic swelling of clays caused by the difference in salt concentrations in connate water in clays and injected water.

*Corresponding author: Chagirov PS, Gubkin Russian State University of Oil and Gas, Russian Federation, Tel: +7 499 507-88-88, Indonesia, E-mail: p.chagirov@gmail.com

Received May 28, 2015; Accepted July 08, 2015; Published July 18, 2015

Citation: Kadet VV, Chagirov PS (2015) Investigation of Cyclic Electrolytic Solutions Injection in Clay-Containing Oil Reservoirs. J Pet Environ Biotechnol 6: 242. doi:10.4172/2157-7463.1000242

Copyright: $\odot 2015$ Kadet $\mathrm{VV}$, et al. This is an open-access article distributed under the terms of the Creative Commons Attribution License, which permits unrestricted use, distribution, and reproduction in any medium, provided the original author and source are credited. 
It's important to take into consideration that at the interface between electrolyte solution and clay mineral osmotic process occurs simultaneously with ion exchange reaction. Studies show [6] that the hydration rate is dependent not only on the type of minerals and concentration disparity between mineral and electrolyte, but also on the ionic composition of the electrolyte solution and connate water of clays. Experimental investigations in [6] give the following dependence

$$
R^{\prime}\left(C_{M e 2}\right)=\frac{R_{0} C_{0}}{C_{M e 1 \Gamma}-C_{0}}\left(\frac{C_{M e 1 \Gamma}}{C_{M e 2}}-1\right)
$$

Where $R_{0}$ - the hydration rate of the mineral in a dilute electrolyte solution, $C_{0}-$ ion concentration in fresh water.

In minerals with a hard crystal structure a chemical adsorption of metal cations of injected water occurs on the mineral surface. It results in substitution of potential-cations by other cations came from injected agent. As a result, an increase in zeta-potential on the surface of clays [7] is observed that leads to an increase in effective viscosity of electrolyte and deviations from linearity of Darcy's law at low pressure gradients [8].

When substituting of the potential-cations in smectite the water molecules penetrate to intermolecular layers of clays as hydration water of cations. Consequently, besides the growth of the zeta-potential on the surface of smectites cation-exchange reactions lead it to swelling.

The concentration of substituting cations, which were included in the connate water of clays from the solution, is determined by the following empirical formula.

$$
C_{M e 2 \Gamma}=C_{M e 1 \Gamma}\left[\left(\frac{C_{M e 1}}{C_{M e 2}}\right) \frac{1}{10 K_{e q} \exp \left(A \sqrt{I_{c}}\left(z_{1}^{2}+z_{2}^{2}\right)\right.}\right]^{-\frac{1}{1-B / R T}}
$$

The value $C_{M e 1}$ is the cation exchange capacity of clays, the most important characteristic of clays in exchange reactions, denominated in mole or milligram-equivalent per $1 \mathrm{~g}$ of clay. The value $C_{M e 1}$ is the quantity of exchangeable cations, which are equivalent of protons in exchange reactions and capable to react with the electrolyte solution. $I_{c}=\frac{1}{2} \sum_{i=1}^{n} C_{M e_{i}} z_{i}$ - ionic strength of the electrolyte; $A$ - characteristic constant of the solvent, the temperature-dependent [9] (for water $A \cong 0.5) ; K_{e q}$ - the equilibrium constant of the cation exchange reaction; $B$ - the value which does not depend on the composition of the solid solution, but depends on the properties of the solution; $R$ universal gas constant. For most montmorillonite clays $C_{M e 1}$ is equal to $1.0 \pm 0.1 \mathrm{mg}$-eq./g [10]. The equilibrium constant is determined by the cation exchange capacity of clays in ion exchange reaction [11].

Figure 1 shows the concentration of substituting cations depending on type of the mineral, its composition and composition of injected solution. In [11] the equilibrium constants $K_{e q}$ used in the calculations have been defined for certain types of clays. Initial parameters: $C_{M e 1}=1 \mathrm{mg}$-eq./g, $C_{M e 1 \Gamma}=10 \mathrm{mg} / \mathrm{g}$.

Compensation of the free energy of the ion occurs by the fact that water molecules make a hydrate shell around the ion. Change of the volume of clay mineral occurs due to the substitution of hydrated ions with different hydration shell. Table 1 shows some of masses and radii of ions in the hydrated state and the Free State [12] Table 1.

By the difference of the radii of hydrated ions $\Delta r_{h}=r_{h 2}-r_{h 1}$ we can assess increase or decrease of clay volume. In case $\Delta r_{h}>0$, an increase of mineral volume occurs. If $\Delta r_{h}<0$ mineral volume decreases.

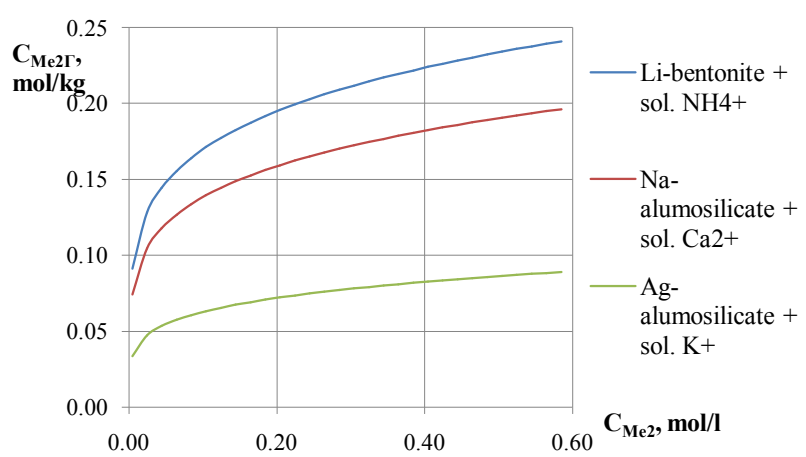

Figure 1: Concentration of substituting cations in clay depending on its concentrationin the solution.

\begin{tabular}{|l|c|c|c|c|c|c|c|c|}
\hline Ion & $\mathbf{L i}^{+}$ & $\mathbf{N a}^{+}$ & $\mathbf{K}^{+}$ & $\mathbf{R b}^{+}$ & $\mathbf{C s}^{+}$ & $\mathbf{N H}_{4}^{+}$ & $\mathbf{F}^{-}$ & $\mathbf{C l}^{-}$ \\
\hline lon radius, $\mathbf{n m}$ & 0.078 & 0.098 & 0.138 & 0.164 & 0.183 & 0.168 & 0.133 & 0.181 \\
\hline $\begin{array}{l}\text { Radius of } \\
\text { hydrated ion, } \mathbf{n m}\end{array}$ & 0.379 & 0.339 & 0.285 & 0.248 & 0.255 & 0.243 & 0.287 & 0.228 \\
\hline
\end{tabular}

Table 1: The ion radii in the free and hydrated state.

\section{Influence of ionic composition of injected agent on the structure of the porous medium}

It is assumed that the clay component is distributed evenly over the entire pore space surface. The authors analyzed the influence of composition of the electrolyte solution, composition and quantity of clay component in rock on the porous medium structure, resulting in a value of the mean radius of the capillary, which takes into account both osmotic and ion exchange processes. Porometrical function and porosity of clay-containing rocks are determined by the mean radius as follows

$$
\begin{aligned}
& f\left(r\left(C_{M e 2}\right)\right)=\frac{1}{r\left(C_{M e 2}\right) a \sqrt{2 \pi}} \exp \left(-\frac{\left(\ln r\left(C_{M e 2}\right)-b\right)^{2}}{2 a^{2}}\right) \\
& m\left(C_{M e 2}\right)=\left(\pi z^{\prime} \int_{0}^{\infty} r\left(C_{M e 2}\right)^{2} f\left(r\left(C_{M e 2}\right)\right) d r\right) / 2 l^{2}
\end{aligned}
$$

Expression (2.1) defines the porometrical function - lognormal probability density function of pore radius - depending on the salt concentration and composition of the liquid being injected. Equation (2.2) determines the porosity of the clay-containing rock depending on the salt concentration and composition of the fluid.

Figure 2 shows porosity depending on the salinity of water and clayiness index.

Figure 3 shows $m(C)$ dependence, calculated using porometrical function (2.2) and the following parameters: $K_{c l}=5 \%$, initial porosity of the core $m_{0}=0.25$, radiuses of hydrated ion are shown in Table 1.

Calculated and analytical results demonstrate that osmotic processes lead to swelling of clays, but ion exchange processes may also lead to clay contraction in reservoir. Calculations show that decrease of porosity can reach $70-80 \%$ when osmotic processes take place. Ion exchange processes lead to a change in porosity in the range of $10-15 \%$.

\section{Analysis of the influence of injected agent mineralization on relative permeabilities}

Consider the displacement of non-wetting liquid by wetting 
Citation: Kadet VV, Chagirov PS (2015) Investigation of Cyclic Electrolytic Solutions Injection in Clay-Containing Oil Reservoirs. J Pet Environ Biotechnol 6: 242. doi:10.4172/2157-7463.1000242

Page 3 of 5

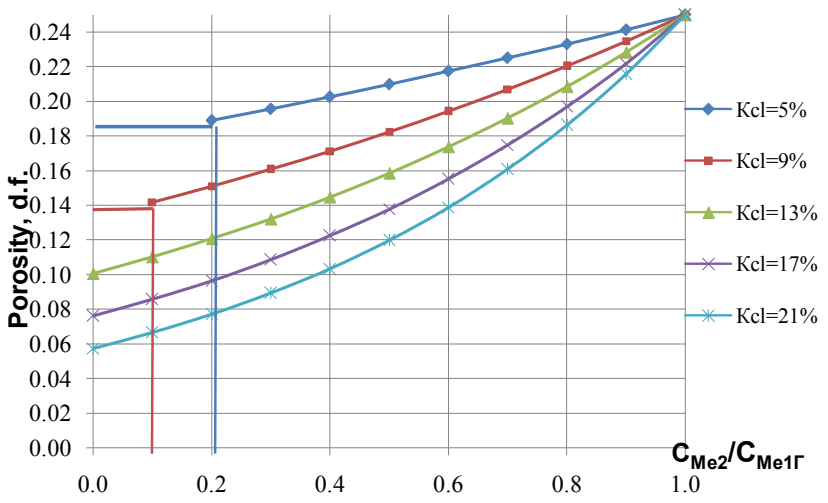

Figure 2: Porosity depending on the concentration and clayiness index.

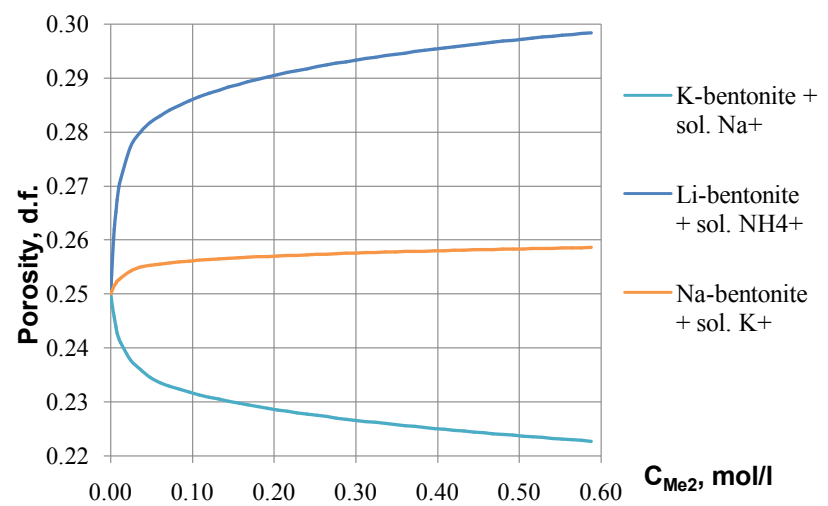

Figure 3: Porosityof clay-containing rocks with different exchange ablecations in the composition depending on the composition and concentration of salts in injected fluid.

liquid, considering liquids as incompressible and viscous fluids. As the displacing fluid penetrates to porous medium, share of the pores filled with water of different composition and salinity than the formation water is restructured due to contraction or swelling of clays, whereas the volume of clay does not change in the pores filled with oil.

In order to obtain analytical relations allowing to calculate and analyze the behavior of relative permeabilities, we use the percolation approach [13]

$$
\begin{aligned}
& k_{i}\left(S, C_{M e 2}\right)=\int_{0}^{r_{i}}\left[\int_{r}^{r_{i}} f_{i}\left(r\left(C_{M e 2}\right), S\right) d r\right]^{r^{\prime}} \frac{f_{i}\left(r\left(C_{M e 2}\right), S\right) d r}{I_{i}\left(r\left(C_{M e 2}\right)\right)}\left\{\int_{0}^{r}\left[\int_{r}^{r} f\left(r\left(C_{M e 2}\right)\right) d r\right]^{r_{0}^{\prime}} \frac{f\left(r\left(C_{M e 2}\right)\right) d r}{I\left(r\left(C_{M e 2}\right)\right)}\right\}^{-1} \\
& I_{i}\left(r\left(C_{M e 2}\right)\right)=\frac{8 \mu}{\pi} \int_{r}^{\infty} r^{-4} f_{i}\left(r\left(C_{M e 2}\right)\right) d r \cdot\left[\int_{r}^{\infty} f_{i}\left(r\left(C_{M e 2}\right)\right) d r\right]^{-1}
\end{aligned}
$$

where $i=1,2$-index of water and oil phase, respectively, $r_{c i}$-critical radiuses for oil and aqueous phases, respectively, $v^{\prime}$ is the index of the correlation radius ( $v^{\prime}=0.85 \pm 0.05$ for three-dimensional lattice).

Figure 4 shows the behavior of the relative permeabilities when changing of salt concentration of electrolyte. In case of osmotic swelling of clays, the same parameters as for determining porosity of the claycontaining rock with $K_{c l}=9 \%$ were used.

Figure 5 shows the effect of ion exchange processes on the relative permeabilities. Calculations were carried out using a concentration of salts in the injected agent $C_{M e 2}=0.5 \mathrm{~mol} / \mathrm{l}$.
Calculations indicate that in case of osmotic processes, when reducing the salt concentration, oil mobility rises and water mobility decreases. In case of the influence of ion exchange processes, an insignificant decrease in permeability to water and growth of permeability to oil is observed.

The model of two-phase fluid flow in porous medium considering capillary pressure and salinity of the aqueous phase

The model of two-phase incompressible fluids flow in porous medium considering capillary pressure is determined by RapoportLease equation $[14,15]$. The choice of this model is due to the fact that when swelling of clays, reservoir gets more fine-pored that requires consideration of capillary forces.

$$
\begin{gathered}
\frac{\partial}{\partial t}\left(m\left(C_{M e 2}\right) S \pm S_{r}\right)+\frac{q}{\rho} \partial_{s} F\left(S, C_{M e 2}\right) \frac{\partial S}{\partial \rho}+\frac{1}{\rho} \frac{\partial}{\partial \rho}\left[\rho M_{2}\left(S, C_{M e 2}\right)\left(\partial_{s} P_{k}\left(S, C_{M e 2}\right) \frac{\partial S}{\partial \rho}\right) F\left(S, C_{M e 2}\right)\right]=0, \\
M_{i}\left(S, C_{M e 2}\right)=\frac{K\left(C_{M e 2}\right) \cdot k_{i}\left(S, C_{M e 2}\right)}{\mu_{i}}, \frac{q}{\rho}=w_{1}+w_{2}, \\
w_{i}=-M_{i}\left(S, C_{M e 2}\right) \frac{\partial P_{i}}{\partial \rho}=-M_{i}\left(S, C_{M e 2}\right) \frac{\Delta P_{i}}{\rho \ln \frac{R_{k}}{r_{w}}} \\
P_{2}-P_{1}=P_{k}\left(S, C_{M e 2}\right)=\chi \sqrt{\frac{m\left(C_{M e 2}\right)}{K\left(C_{M e 2}\right)}} J(S)
\end{gathered}
$$

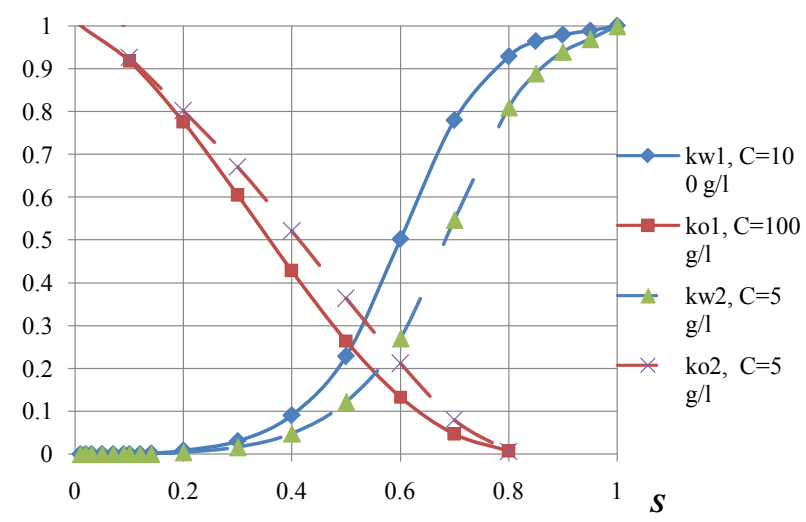

Figure 4: Relative permeabilities for oil and water depending on concentrations of salts in the injected water in case of osmotics welling of clays.

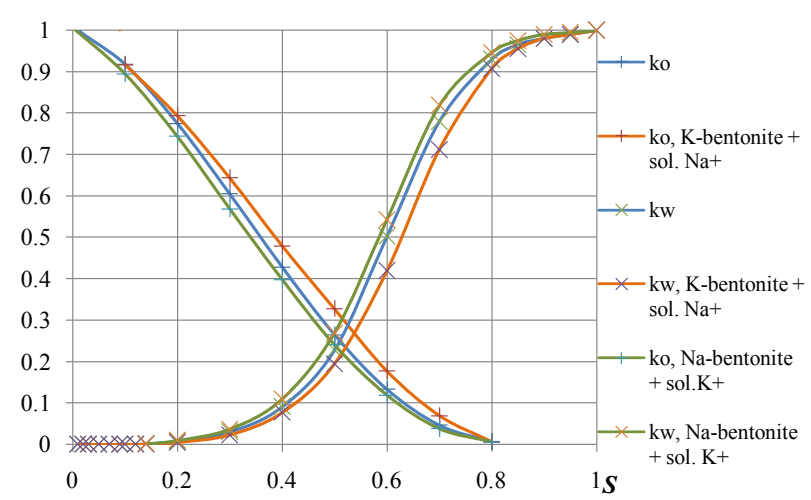

Figure 5: Relative permeabilities to water and oil in the clay-containing rocks of different composition of exchangeable cations, depending on the composition of saltsin the injected fluid. 
Citation: Kadet VV, Chagirov PS (2015) Investigation of Cyclic Electrolytic Solutions Injection in Clay-Containing Oil Reservoirs. J Pet Environ Biotechnol 6: 242. doi:10.4172/2157-7463.1000242

where $S_{r}$ - share of adsorbed water in rock, $F\left(S, C_{M e 2}\right)$ - Buckley-Leverett function, $q$ - unit discharge, $P_{k}\left(S, C_{M e 2}\right)$ - capillary pressure function, $M_{i}\left(S, C_{M e 2}\right)$ - phase mobility, $R_{k}$ - radius of the reservoir external boundary, $r_{w}$ - well radius.

Salt transfer in fluid flow is described by diffusion-convective equation

$$
\frac{\partial m\left(C_{M e 2}\right) S C_{M e 2}}{\partial t}+\frac{\partial w_{1} C_{M e 2}}{\partial \rho}=D^{*} \frac{1}{\rho} \frac{\partial}{\partial \rho}\left(\rho \frac{\partial C_{M e 2}}{\partial \rho}\right)-R^{\prime}\left(C_{M e 2}\right)
$$

where $w_{1}$ - the filtration rate of the aqueous phase, $D^{*}$ - coefficient taking into account diffusion and hydrodynamic dispersion.

As a result, we obtain a system of two differential equations for $C_{M e 2}$ and $S$. The following parameters are set for the calculations: pressure drop - $15 \mathrm{MPa}$; initial porosity -0.2 ; absolute permeability $-5 \cdot 10^{-2} \mu m^{2}$; viscosity of water $-0.6 \mathrm{mPas}$; oil viscosity $-5 \mathrm{mPas}$. It's assumed that reservoir contains bentonite, $K_{c l}=5 \%$, formation water contains ions $K^{+}$, $\mathrm{C}_{K}-0.8 \mathrm{~mol} / \mathrm{l}$. Injected water contains $\mathrm{Na}^{+}, \mathrm{C}_{\mathrm{Na}}-0.8 \mathrm{~mol} / \mathrm{l}$. Reservoir size: formation thickness $-10 \mathrm{~m}$, external boundary of reservoir -500 $m$, well radius $-0.05 m$.

Initial conditions: $S(\rho, 0)=0.2, C_{M e 2}(\rho, 0)=C_{K}$. Boundary conditions: $S\left(R_{k}, t\right)=0.8, \partial S\left(R_{k}, t\right)=0, \partial C_{M e 2}\left(R_{k}, t\right)=0 ; C_{M e 2}\left(R_{k}, t\right)=10^{-2} C_{K}$ in case of low salinity water injection; $C_{M e 2}\left(R_{k}, t\right)=C_{N a}$ in case of electrolytic solution injection Figure 6 shows oil recovery depending on development time.

Results demonstrate that electrolytic solution injection increases oil recovery by $6 \%$ in comparison with formation water injection, whilst low salinity water injection increases oil recovery by $15 \%$. However, it's been shown in [4] that fresh or low salinity water injection leads to destruction of clay in reservoir which means that absolute permeability drops while injecting that solutions. As high salinity electrolytic solutions cause swelling of clays without destruction, high salinity electrolytic solutions is recommended as displacing agent.

Results shown on Figure 6 are confirmed by experimental data $[1,2,3]$.

\section{Modeling of cyclic electrolytic solutions injection in clay- containing oil reservoirs}

Let's consider the influence of injection frequency of electrolytic solutions on oil recovery. The purpose of this consideration is possibility to save salts.

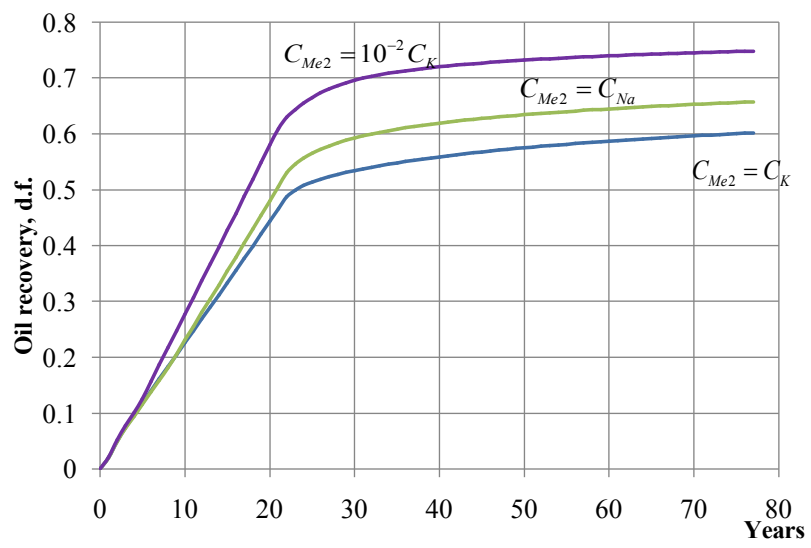

Figure 6: Oil recovery depending on development time in case of low salinity waterflooding and electrolytic solution waterflooding.

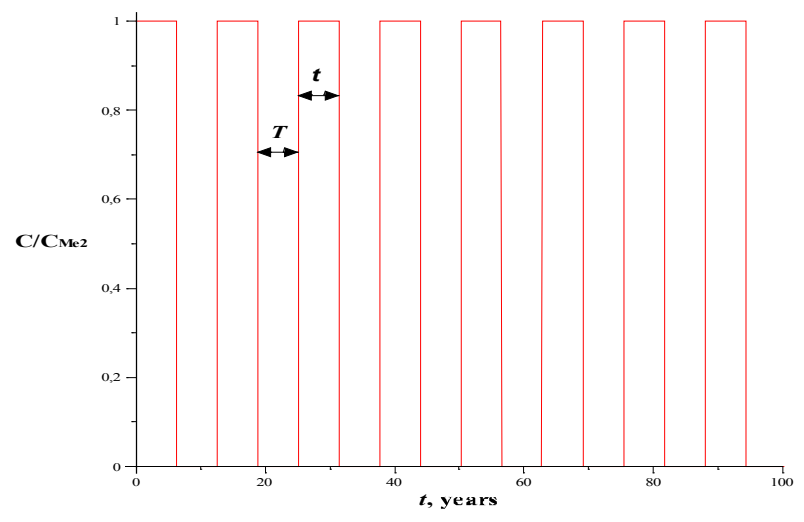

Figure 7: Concentration of electrolyte on external boundary depending on development time, $T$-formation water injection time, $T$-electrolytic solution injection time.

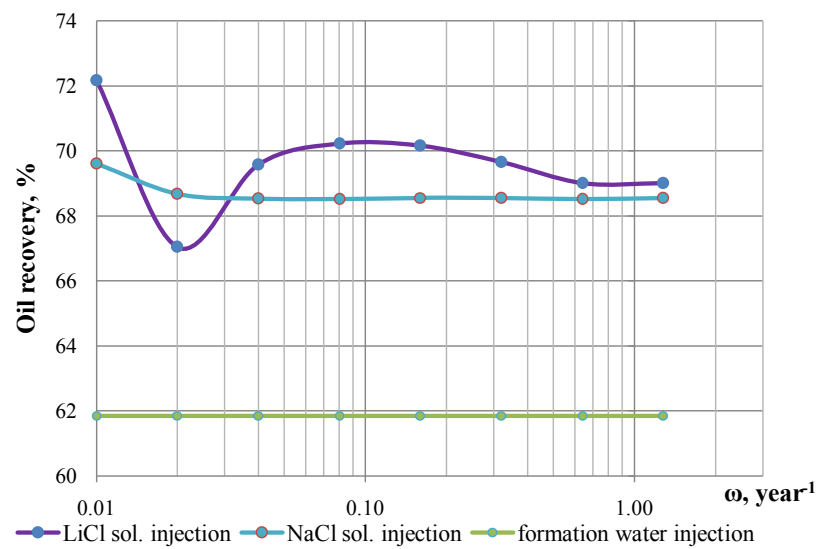

Figure 8: Oil recovery depending on injection frequency of electrolytic solutions, $\omega=T^{1}$.

The problem is formulated as follows: we alternately inject formation water and electrolytic solution into the oil-saturated and clay-containing reservoir. Initial condition are the same as in the 4-th paragraph. Boundary condition is shown on Figure 7 and described by the following equation

$$
C(t, \tau, T)=\frac{C_{M e 2}}{2}+\frac{C_{M e 2}}{2} \sum_{n} \frac{4}{\pi n} \sin \left(2 \pi n \frac{\tau}{2(T-\tau)}\right) \cos \left(2 \pi n \frac{t}{T-\tau}\right)
$$

Calculations are shown on Figure 8.

It's shown that cyclic injection of electrolytic solutions with highly active metal ions (i.e. $\mathrm{LiCl}$ ) allows to save $50 \%$ of salts and increases oil recovery by $8.5 \%$ (i.e. $\mathrm{LiCl}$ ) and $7 \%$ (i.e. $\mathrm{NaCl}$ ) in comparison with formation water injection.

\section{Conclusion}

The analysis of osmotic and ion exchange processes in claycontaining porous medium allowed to determine the reasons for the growth of oil recovery when changing the hydrochemical regime of reservoir. It is shown that osmotic process at the interface between rock and displacing agent lead to an increase in relative permeability to oil and decrease in relative permeability to water. The growth in relative permeability to water and its reduction to oil is caused by ion exchange 
Citation: Kadet VV, Chagirov PS (2015) Investigation of Cyclic Electrolytic Solutions Injection in Clay-Containing Oil Reservoirs. J Pet Environ Biotechnol 6: 242. doi:10.4172/2157-7463.1000242

processes leading to clay contraction. This is why swelling of clay leads to enhanced oil recovery - relative permeability to oil increases when injecting electrolytic solutions with highly active metal ions.

Due to the osmotic swelling of clays porosity may decrease three times, whereas due to the ion-exchange process change of porosity is about $10 \%$. This is also the reason why oil recovery increases. When porosity of reservoir decreases, the reservoir works like a sponge, squeezing out oil from reservoir.

Calculations show that electrolytic solutions injection increases oil recovery up to $10 \%$ in comparison with formation water injection. Cyclic injection of electrolytic solutions with highly active metal ions (i.e. $\mathrm{LiCl}$ ) allows to save $50 \%$ of salts and increases oil recovery by $8.5 \%$ (i.e. $\mathrm{LiCl}$ ) and $7 \%$ (i.e. $\mathrm{NaCl}$ ) in comparison with formation water injection. The created model allows evaluating the influence of injected agent composition on technological performance of claycontaining reservoirs. Calculated results are qualitatively matched with experimental data $[1,2,3]$.

\section{References}

1. Tang GQ, Morrow NR (1999) Influence of brine composition and fines migration on crude oil/brine/rock interactions and oil recovery. Journal of Petroleum Science and Engineering 24: 99-111.

2. Morrow N, Buckley J (2011) Improved oil recovery by Low-Salinity Waterflooding Journal of Petroleum Technology 63: 106-112.

3. Austad T, Rezaeidoust A, Puntervold T (2010) Chemical Mechanism of Low Salinity Water Flooding in Sandstone Reservoirs. SPE Improved Oil Recovery Symposium Tulsa, Oklahoma, USA: Society of Petroleum Engineers.
4. Stupochenko VE (1981) Vlijanie glinistosti kollektora na polnotu vytesnenija nefti vodoj. Geological and geophysical foundations of recovery factor 228: 59-79.

5. Hanin AA (1969) Porody i kollektory nefti i gaza i ih izuchenie. Publishing House «Nedra»: 140-141.

6. Ulyasheva NM, Ivenina IV (2010) Vlijanie ionnoj sily rastvora na skorost uvlazhnenija glinistyh porod. Construction of oil and gas wells on land and at sea $4: 28-30$.

7. Kadet VV, Koryuzlov AS (2008) Electro kinetic Effects on Pressure-driven Flow in Porous Media. 11 th European Conference on the Mathematics of Oil Recovery.

8. Kadet VV, Chagirov PS (2012) Alternative to Darcy's Law for the Description of the Newtonian Fluid Flow in Porous Media. $13^{\text {th }}$ European Conference on the Mathematics of Oil Recovery.

9. Manov GG, Vates RG, Hamer WJ, Asree SF (1943) Journal of American Chemical Society 65: 1765

10. Carroll D (1959) lon exchange in clays and other minerals, Bull. Geological Society of America 70: 754.

11. Walton HF (1949) Ion exchange equilibria, in Ion Exchange Theory and Practice, Nachod FC (Ed.), New York, Academic.

12. Baldanov MM, Baldanova DM, Zhigzhitova SB, Tanganov BB (2006) On the problem of the radii of hydrated ions. DAN VS Russia 2: 32-34.

13. Kadet VV (2008) Methods of percolation theory in the underground fluid mechanics. Tsentr Lit Nefte Gaz: 96.

14. Selyakov VI, Kadet VV (1996) Percolation Models for Transport in Porous Media. Kluwer Academic Publishers: 241

15. Kadet VV, Chagirov PS, Electrokinetic Mechanism of Low Salinity Water Flooding and Modeling of Oil Displacement in Clay Reservoirs. $14^{\text {th }}$ European Conference on the Mathematics of Oil Recovery. 